\title{
A NOTE ON SUBGROUPS IN A DIVISION RING THAT ARE LEFT ALGEBRAIC OVER A DIVISION SUBRING
}

\author{
BUI XUAN HAI, VU MAI TRANG, AND MAI HOANG BIEN
}

\begin{abstract}
Let $D$ be a division ring with center $F$ and $K$ a division subring of $D$. In this paper, we show that a non-central normal subgroup $N$ of the multiplicative group $D^{*}$ is left algebraic over $K$ if and only if so is $D$ provided $F$ is uncountable and contained in $K$. Also, if $K$ is a field and the $n$-th derived subgroup $D^{(n)}$ of $D^{*}$ is left algebraic of bounded degree $d$ over $K$, then $\operatorname{dim}_{F} D \leq d^{2}$.
\end{abstract}

\section{INTRODUCTION}

Let $D$ be a division ring with center $F$ and $K$ a division subring of $D$. Recall that an element $a \in D$ is left algebraic over $K$ if there exist $a_{0}, a_{1}, \cdots, a_{n} \in K$ not all are zeros such that $a_{0}+a_{1} a+\cdots+a_{n} a^{n}=0$, or equivalently, there exists a non-zero polynomial $f(t) \in K[t]$ whose coefficients are written on the left such that $f(a)=0$. Here, we have to emphasize the convention that for polynomials over a ring $R$ (commutative or not), a polynomial $f(t) \in R[t]$ can be written in two ways such as

$$
f(t)=\sum_{\text {finite }} a_{i} t^{i}=\sum_{\text {finite }} t^{i} a_{i}
$$

However, if $a \in S$, where $S$ is a ring containing $R$, the substitution functions may give the different values, i.e., we may have

$$
\sum_{\text {finite }} a_{i} a^{i} \neq \sum_{\text {finite }} a^{i} a_{i}
$$

In this paper, we always mean

$$
f(a)=\sum_{\text {finite }} a_{i} a^{i}
$$

and then $a$ is called a right root of $f(t)$. A left root of $f(t)$ and a right algebraic element are defined similarly. A subset $S$ is called left algebraic (resp., right algebraic) over $K$ if every element in $S$ is left algebraic (resp., right algebraic) over $K$. If $K$ is central, that is, $K \subseteq F$, then the right algebraicity coincides with the left one. However, if $K$ is not central, then there are division rings $K \subseteq D$ such that $D$ is left algebraic but not right algebraic over $K$. Observe that with division rings $K \subseteq D$ which were presented by Cohn in [6], we can show that the division ring $D$ is left algebraic but not right algebraic over $K$. In Section 2, we give a natural and simple example of division rings $K \subseteq D$ such that $D^{*}$ contains a proper normal

Key words and phrases. right (left) algebraic, derived subgroups, normal subgroups 2010 Mathematics Subject Classification. 16K20, 16K40, $16 \mathrm{R} 20$.

The first and the third authors were funded by Vietnam National University HoChiMinh City (VNUHCM) under grant number C2018-18-03. 
subgroup $N$ which is left algebraic but not right algebraic over $K$. In the case when $K$ is a subfield of $D$, it is not known whether $D$ is left algebraic over $K$ provided it is right algebraic over $K$ (see [4, Page 1610]).

The notion of one-sided (right or left) algebraicity has been introduced to study polynomials over division rings. For example, 9] (see Chapter 7) is one of the oldest books mentioning this notion. Some special cases of one-sided algebraicity were studied by several authors. For instance, C. Faith [7] investigated division rings that are radical over their division subrings, P. M. Cohn and A. H. Schofield 6. 13. studied the left and right dimensions of a division ring $D$ over its division subring $K$. Recently, division rings whose elements are algebraic (left or right) over their division subrings have been received a considerable attention (see, for example [1, 3, 4, 5, 8, and references therein).

In this note, firstly, we are interested in the question whether the algebraicity of some subset in a division ring $D$ over its proper division subring $K$ may entail the algebraicity of whole $D$. More exactly, we pose the following conjecture to study.

Conjecture 1. Let $D$ be a division ring with center $F, K$ a division subring of $D$ containing $F$, and $N$ a subnormal subgroup of $D^{*}$. If $N$ is non-central, then $N$ is left algebraic (resp., right algebraic) over $K$ if and only if so is $D$.

In Section 3 (see Theorem 3.3), we give the answer to this conjecture in the case when $N$ is a normal subgroup of $D^{*}$ and $F$ is uncountable.

Secondly, in Section 4 we study a division ring $D$ whose $n$-th derived subgroup $D^{(n)}$ of $D^{*}$ is left algebraic of bounded degree over some subfield (recall that the $n$-th derived subgroup $D^{(n)}$ of $D^{*}$ is defined as follow: $D^{(1)}=D^{\prime}$ is the commutator subgroup of $D^{*}$ and $D^{(n)}$ is the commutator subgroup of $D^{(n-1)}$ for $\left.n>1\right)$. Note that in 4, it was proved that if $D$ is a division ring with center $F$ and there exists a subfield $K$ of $D$ such that $D$ is left algebraic over $K$ of bounded degree $d$, then $\operatorname{dim}_{F} D \leq d^{2}$. This result was extended for the commutator subgroup $D^{\prime}$ instead of $D$ in [1, Theorem 17]. The result we get in Theorem 4.4 generalizes this fact by considering $D^{(n)}$ for an arbitrary $n \geq 1$ instead of $D^{\prime}$.

\section{ExAmple}

In this section, we give an example of division rings $K \subseteq D$ such that $D^{*}$ contains a proper normal subgroup $N$ which is left algebraic but not right algebraic over $K$.

Let $F$ be a field with an endomorphism $\sigma$ and $t$ an indeterminate. We denote by $F((t, \sigma))=\left\{\sum_{i=n}^{\infty} a_{i} t^{i} \mid a_{i} \in F, n \in \mathbb{Z}\right\}$ the ring of Laurent skew series in $t$ over $F$ with respect to $\sigma$ in which the addition is defined as usual, and the multiplication is an extension of the rule $t a=\sigma(a) t$. In general, $F((t, \sigma))$ is not a division ring (for example, if $\sigma$ is not injective, then there exists $a \in F^{*}$ such that $\sigma(a)=0$, so $t a=\sigma(a) t=0)$. However, if $\sigma$ is injective, then $F((t, \sigma))$ is a division ring [10, Example 1.7]. For $\alpha=\sum_{i=n}^{\infty} a_{i} t^{i}$, the lowest power appearing in $\alpha$ is denoted by $\operatorname{degmin}(\alpha)$. That is, $\operatorname{degmin}(\alpha)=\min \left\{i \mid a_{i} \neq 0\right\}$.

Now let $k$ be a field of characteristic 0 and $\left\{x_{0}, x_{1}, \cdots\right\}$ a countable set of commuting indeterminates. Consider the field of fractions $F=k\left(x_{0}, x_{1}, \ldots\right)$ of the polynomial ring $k\left[x_{0}, x_{1}, \ldots\right]$, and the endomorphism $\sigma: F \rightarrow F$ defined by $\sigma\left(x_{i}\right)=x_{i+1}$ for $i \in \mathbb{N}$. Then, we have the following easy lemma. 
Lemma 2.1. Let $F=k\left(x_{0}, x_{1}, \ldots\right)$ and $\sigma: F \rightarrow F$ be defined above. Then $D=F((t, \sigma))$ is a division ring and

$$
\operatorname{degmin}(\alpha \cdot \beta)=\operatorname{degmin}(\alpha)+\operatorname{degmin}(\beta)
$$

for every $\alpha, \beta \in D$. In particular, $\operatorname{degmin}\left(\alpha^{-1}\right)=-\operatorname{degmin}(\alpha)$ for every $\alpha \in D^{*}$.

Proof. The proof of the first conclusion is essentially due to that of 10, Example 1.7 and Proposition 14.2]. The proof of the second one is elementary by the fact that $\sigma$ is injective.

Now, we are ready to give an example we have mentioned in the begining of this section.

Example 2.2. Let $D=F((t, \sigma))$ be as in Lemma 2.1 and consider the following subset in $D$ :

$$
K=F\left(\left(t^{2}, \sigma\right)\right)=\left\{\sum_{i=n}^{\infty} a_{i} t^{2 i} \mid n \in \mathbb{Z}, a_{i} \in F\right\} .
$$

It is easy to see that $K$ is a division subring of $D$. It is obvious that if $\alpha=\sum_{i=n}^{\infty} a_{i} t^{i} \in$ $D$, then

$$
\alpha=\sum_{i=2 j \geq n} a_{i} t^{i}+\sum_{i=2 j+1 \geq n} a_{i} t^{i},
$$

that is, $\alpha=\alpha_{1}+\alpha_{2} t$, where $\alpha_{1}, \alpha_{2} \in K$. Hence, $\{1, t\}$ is a basis of the left vector space $D$ over $K$, which implies that the dimension of the left vector space $D$ over $K$ is 2 . Hence, every element of $D$ is left algebraic of degree $\leq 2$ over $K$. Now, let $N=\left\{\alpha \in D^{*} \mid \operatorname{degmin}(\alpha)=0\right\}$. We claim that $N$ is a proper normal subgroup of $D^{*}$. Indeed, it is trivial that $N \neq D^{*}$. For $\alpha, \beta \in N$, the condition $\operatorname{degmin}(\alpha)=\operatorname{degmin}(\beta)=0$ implies $\operatorname{degmin}(\alpha \beta)=\operatorname{degmin}(\alpha)+\operatorname{degmin}(\beta)=0$ and $\operatorname{degmin}\left(\alpha^{-1}\right)=-\operatorname{degmin}(\alpha)=0$. Therefore, $\alpha \beta, \alpha^{-1} \in N$, which shows that $N$ is subgroup of $D^{*}$. Assume that $\alpha \in N$ and $\beta \in D^{*}$. Then,

$\operatorname{degmin}\left(\beta^{-1} \alpha \beta\right)=\operatorname{degmin}\left(\beta^{-1}\right)+\operatorname{degmin}(\alpha)+\operatorname{degmin}(\beta)$

$$
=-\operatorname{degmin}(\beta)+\operatorname{degmin}(\beta)=0 \text {. }
$$

As a corollary, $\beta^{-1} \alpha \beta \in N$, so $N$ is normal in $D^{*}$.

Consider the element $x_{0}+t \in N$. To finish example, we will show that $x_{0}+t$ is not right algebraic over $K$. Suppose that there exist $h_{0}\left(t^{2}\right), h_{1}\left(t^{2}\right), \cdots, h_{n}\left(t^{2}\right) \in K$ such that $h_{n}\left(t^{2}\right) \neq 0$ and

$$
h_{0}\left(t^{2}\right)+\left(x_{0}+t\right) h_{1}\left(t^{2}\right)+\cdots+\left(x_{0}+t\right)^{n} h_{n}\left(t^{2}\right)=0 .
$$

We seek a contradiction. Indeed, observe that, after expanding, $\left(x_{0}+t\right)^{i}$ is written as a linear sum of term $x_{0}^{m_{0}} x_{1}^{m_{1}} \cdots x_{i-1}^{m_{i-1}} t^{m}$ over $\mathbb{Z}$, so one sees that the term $x_{0}^{n-1} t$ appears in $\left(x_{0}+t\right)^{n}$ but does not in $\left(x_{0}+t\right)^{i}$ with $i<n$. Moreover, all powers of $t$ appearing in $h_{0}\left(t^{2}\right), \ldots, h_{n-1}\left(t^{2}\right)$ is even, so $x_{0}^{n-1} t h_{n}\left(t^{2}\right)=0$, equivalently, $h_{n}\left(t^{2}\right)=0$, a contradiction. Thus, $x_{0}+t$ is not right algebraic over $K$.

\section{Left algebraic NORMal SUbGROUPS IN A DIVISION RING}

For a division ring $D$ and its division subring $K,{ }_{K} D$ and $D_{K}$ denote the left and right vector space over $K$ respectively. In this section, we give the affirmative answer to Conjecture 1 in the case when $F$ is uncountable and $N$ is a non-central normal subgroup of $D^{*}$.

We need some lemmas. 
Lemma 3.1. 14, Page 440] Let $D$ be a division ring with center $F$ and $N$ a normal subgroup of $D^{*}$. If $N$ is non-central, then $C_{D}(N)=F$.

Lemma 3.2. Let $D$ be a division ring with center $F, K$ a division subring of $D$ containing $F$ and $a$ an element of $D$. Assume that $\alpha_{1}, \alpha_{2}, \cdots, \alpha_{n}$ are distinct elements in $F$ such that all elements $a-\alpha_{i}$ are non-zeros. Then, either $a$ is left (resp. right) algebraic over $K$ or the set $\left\{\left(a-\alpha_{i}\right)^{-1} \mid i=1,2, \cdots, n\right\}$ is left (resp. right) linearly independent over $K$.

Proof. It is enough to prove the lemma for the case of left algebraicity since the case of right algebraicity is similar. Assume that $a$ is not left algebraic over $K$ and

$$
\beta_{1}\left(a-\alpha_{1}\right)^{-1}+\beta_{2}\left(a-\alpha_{2}\right)^{-1}+\cdots \beta_{n}\left(a-\alpha_{n}\right)^{-1}=0
$$

for some $\beta_{i} \in K$. Consider the polynomials

$$
f(t)=\left(t-\alpha_{1}\right)\left(t-\alpha_{2}\right) \cdots\left(t-\alpha_{n}\right) \in F[t] \subseteq K[t]
$$

and $f_{i}(t)=f(t) /\left(t-\alpha_{i}\right)$ for $1 \leq i \leq n$. Multiplying both sides of (1) on the right by $f(a)$, we get $\beta_{1} f_{1}(a)+\beta_{2} f_{2}(a)+\cdots+\beta_{n} f_{n}(a)=0$. This shows that $a$ is a right root of the polynomial $g(t)=\beta_{1} f_{1}(t)+\beta_{2} f_{2}(t)+\cdots+\beta_{n} f_{n}(t)$, so $g(t) \equiv 0$ because $a$ is not left algebraic over $K$. Then, for every $1 \leq i \leq n$, we have $0=g\left(\alpha_{i}\right)=\beta_{i} f_{i}\left(\alpha_{i}\right)$. Therefore, $\beta_{i}=0$ for all $i$. Hence, the set $\left\{\left(a-\alpha_{i}\right)^{-1} \mid i=1,2, \cdots, n\right\}$ is left linearly independent over $K$.

Note that the special case of Lemma 3.2 when $K=F$ was considered in 12 , Proposition 5.2.21].

Theorem 3.3. Let $D$ be a division ring with uncountable center $F, K$ a division subring of $D$ containing $F$ and $N$ a normal subgroup of $D^{*}$. If $N$ is non-central, then $N$ is left algebraic (resp., right algebraic) over $K$ if and only if so is $D$.

Proof. We show that the theorem is true for the left case since the proof for the right case is similar. Thus, assume that $N$ is a non-central normal subgroup of $D^{*}$ which is left algebraic over $K$. For any $a \in D$, we have to prove that $a$ is left algebraic over $K$. If $a \in C_{D}(N)$, then by Lemma 3.1, $a \in F \subseteq K$, and there is nothing to prove. Now, assume that $a \notin C_{D}(N)$. Take $b \in N$ such that $d=b a-a b \neq 0$. For every $\alpha \in F$, one has

$$
\begin{gathered}
d=b a-a b=b(a+\alpha)-(a+\alpha) b \\
=b(a+\alpha)\left(1+(a+\alpha)^{-1} b^{-1}(a+\alpha) b\right)=b(a+\alpha)(1+c),
\end{gathered}
$$

where $c=(a+\alpha)^{-1} b^{-1}(a+\alpha) b \in N$. Since $c$ is left algebraic over $K$ and $c+1 \neq 0$, the element $(c+1)^{-1}$ is left algebraic over $K$. Consequently, $d^{-1} b(a+\alpha)=(c+1)^{-1}$ is left algebraic over $K$. Hence, there exist $\beta_{1}, \beta_{2}, \cdots, \beta_{n} \in K$ such that

$$
\beta_{n}\left(d^{-1} b(a+\alpha)\right)^{n}+\beta_{n-1}\left(d^{-1} b(a+\alpha)\right)^{n-1}+\cdots+\beta_{1}\left(d^{-1} b(a+\alpha)\right)+1=0 .
$$

We can write this equality as follows:

$$
1=\left(-\beta_{n}\left(d^{-1} b(a+\alpha)\right)^{n-1}-\cdots-\beta_{1}\left(d^{-1} b\right)\right)(a+\alpha) .
$$

Therefore, $(a+\alpha)^{-1}=-\beta_{n}\left(d^{-1} b(a+\alpha)\right)^{n-1}-\cdots-\beta_{1} d^{-1} b$ is in the left vector $K$-subspace $W$, which is generated by the subgroup $\langle a, b, d\rangle$ of $D^{*}$ generated by $a, b, d$. Since $\langle a, b, d\rangle$ is a finitely generated subgroup, the cardinality of the basis of $W$ over $K$ is countable. Observe that $F$ is uncountable, so is the set

$$
\left\{(a+\alpha)^{-1} \mid \alpha \in F\right\} \text {. }
$$


As a corollary, the set $\left\{(a+\alpha)^{-1} \mid \alpha \in F\right\}$ is left linearly dependent over $K$. In view of Lemma 3.2, $a$ is left algebraic over $K$.

The following corollary gives the affirmative answer to [11, Problem 13] in the case of uncountable center $F$ of $D$ and $N$ is normal in $D^{*}$.

Corollary 3.4. Let $D$ be a division ring with uncountable $F$. Assume that $N$ is a non-central normal subgroup of $D^{*}$. If $N$ is algebraic over $F$, then so is $D$.

\section{LEFT ALGEBRAIC $n$-TH DERIVED SUbGROUP OF BOUNDED DEGREE}

Let $D$ be a division ring and $K$ a subfield of $D$. In this section, we prove that if for some integer $n \geq 1$, the $n$-th derived subgroup $D^{(n)}$ is left algebraic of bounded degree $d$ over $K$, then $\operatorname{dim}_{F} D \leq d^{2}$

The proof of the following lemma is elementary, so we omit it.

Lemma 4.1. Let $D$ be a division ring, $K$ a division subring and $x$ an element in $D$. The following conditions are equivalent.

(1) The element $x$ is left algebraic (resp., right algebraic) over $K$ of degree $d$.

(2) $d$ is the largest integer such that $\left\{x^{n} \mid n=1,2, \cdots, d-1\right\}$ is a left (resp., right) linearly independent set in ${ }_{K} D$ (resp., $D_{K}$ ).

(3) $d$ is the largest integer such that the sum $\sum_{i=0}^{d-1} K x^{i}$ (resp., $\sum_{i=0}^{d-1} x^{i} K$ ) is a direct sum in ${ }_{K} D$ (resp., $\left.D_{K}\right)$.

Lemma 4.2. Let $D$ be a division ring with infinite center, $N$ a non-central normal subgroup of $D^{*}$ and $K$ a subfield of $D$. If $N$ is left (or right) algebraic over $K$ of bounded degree, then $D$ is centrally finite, that is, $D$ is a finite dimensional vector space over its center.

Proof. Since $N$ is non-central, there exists $a \in N \backslash F$. For every $x \in D^{*}$, one has $a x a^{-1} x^{-1}=a\left(x a^{-1} x^{-1}\right) \in N$, so $a x a^{-1} x^{-1}$ is left (resp., right) algebraic of bounded degree over $K$. By [1, Theorem 11], $D$ is centrally finite.

Corollary 4.3. Let $D$ be a division ring with infinite center. For any positive integer $n$, if the $n$-the derived subgroup $D^{(n)}$ is left (or right) algebraic of bounded degree over some subfield of $D$, then $D$ is centrally finite.

Proof. If $D^{(n)}$ is central, then $D^{*}$ is solvable, so $D$ is a field by 14, 14.4.4, Page 440]. Hence, we can assume that $D^{(n)}$ is non-central. Since $D^{(n)}$ is normal in $D^{*}$, by Lemma 4.2 $D$ is centrally finite.

The following theorem extends [4, Theorem 1.3] and [1, Theorem 17].

Theorem 4.4. Let $D$ be a division ring with infinite center $F, K$ a subfield of $D$ and $n$ be a positive integer. If the $n$-th derived subgroup $D^{(n)}$ is left (or right) algebraic of bounded degree $d$ over $K$, then $\operatorname{dim}_{F} D \leq d^{2}$.

Proof. We prove the theorem for the left case since the right case is similar. Without loss of generality, we assume that $K$ is a maximal subfield of $D$. According to Corollary 4.3, $\operatorname{dim}_{F} D=m^{2}<\infty$. We must show that $m \leq d$. By 2 , Theorem 7], there exists $x \in D^{(n)}$ such that $L=F(x)$ is a maximal subfield of $D$. It is well known that $\operatorname{dim}_{F} L=m$ (or see [10, Proposition 15.7 and Theorem 15.8]). One has that $D$ is a left $D \otimes_{F} L$-module in which the operator is defined by $\left(\alpha \otimes x^{i}\right) \beta=\alpha \beta x^{i}$ for every $\alpha, \beta \in D$ and $i \in \mathbb{N}$. Observe that $D \otimes_{F} L$ is simple, so $D$ is faithful. 
On the other side, $D$ may be considered as a left $K$-space. Now, consider $T \in$ End $_{K} D$ which is defined by $T(\alpha)=\alpha x$ for every $\alpha \in D$. We claim that the set $\left\{T^{i} \mid i=0,1, \cdots, m-1\right\}$ is left linearly independent over $K$. Indeed, assume that $\sum_{i=0}^{m-1} c_{i} T^{i}=0$ for some $c_{0}, c_{1}, \cdots, c_{m-1} \in K$. Then, for every $\alpha \in D$,

$$
0=\left(\sum_{i=0}^{m-1} c_{i} T^{i}\right)(\alpha)=\sum_{i=0}^{m-1} c_{i} \alpha x^{i}=\left(\sum_{i=0}^{m-1} c_{i} \otimes x^{i}\right) \alpha
$$

Observe that $D$ is faithful, so $\sum_{i=0}^{m-1} c_{i} \otimes x^{i}=0$, which implies that

$$
c_{0}=c_{1}=\cdots=c_{m-1}=0 .
$$

The claim is proved. The next claim is that there exists $y \in D$ such that $K y+$ $K T(y)+\cdots+K T^{m-1}(y)$ is a direct sum. Indeed, let $t$ be an indeterminate and $K[t]$ be the polynomial ring in $t$ over $K$. Then, we can consider $D$ as left $K[t]$-module with operator defined by the rule $f(t) . \alpha=f(T)(\alpha)$ for every $\alpha \in D$ and $f(t) \in K[t]$. Since $\operatorname{dim}_{K} D<\infty$, there exists a non-zero element $g(t) \in K[t]$ such that $g(T)=0$. Hence, for every $\alpha \in D$, one has $g(t) \alpha=g(T)(\alpha)=0$, so it follows that $D$ is torsion as left $K[t]$-module. Moreover, it is obvious that $D$ is finitely generated as left $K$ space, so is $D$ as a left $K[t]$-module. Therefore, $D$ is torsion finitely generated as a left module over a PID. Hence, there exist $f_{1}(t), f_{2}(t), \cdots, f_{\ell}(t) \in K[t]$ such that

$$
\left\langle f_{1}(t)\right\rangle \supseteq\left\langle f_{2}(t)\right\rangle \supseteq \cdots \supseteq\left\langle f_{\ell}(t)\right\rangle
$$

and an isomorphism

$$
\phi: K[t] /\left\langle f_{1}(t)\right\rangle \oplus K[t] /\left\langle f_{2}(t)\right\rangle \oplus \cdots \oplus K[t] /\left\langle f_{\ell}(t)\right\rangle \rightarrow D,
$$

where $\langle f(t)\rangle$ denotes the ideal of $K[t]$ generated by some element $f(t) \in K[t]$. Put $y=\phi\left(1+\left\langle f_{\ell}(t)\right\rangle\right) \in D$. We will show that $y$ is the element we need to find. Indeed, assume that $f(t)=c_{0}+c_{1} t+\cdots+c_{m-1} t^{m-1} \in K[t]$ such that $f(T)(y)=0$. Then, $f(t) y=0$, equivalently, $f(t) \in \operatorname{ann}_{K[t]} y$. Observe that $\langle y\rangle \cong R /\left\langle f_{\ell}(t)\right\rangle$ and by direct calculation, one has

$$
\operatorname{ann}_{K[t]}(D)=\bigcap_{i=1}^{\ell} \operatorname{ann}_{K[t]} K[t] /\left\langle f_{i}(t)\right\rangle=\bigcap_{i=1}^{\ell}\left\langle f_{i}(t)\right\rangle=\operatorname{ann}_{K[t]} y .
$$

Hence, $f(t) \in \operatorname{ann}_{K[t]} y=\operatorname{ann}_{K[t]} D$. As a corollary, $f(T)(\alpha)=0$ for every $\alpha \in$ $D$ which contradicts to the fact that $\left\{T^{i} \mid i=0,1, \cdots, m-1\right\}$ is left linearly independent over $K$. Therefore, the claim is proved. Put $u=y x y^{-1}$. Then,

$$
K+K u+\cdots+K u^{m-1}=\left(K y+K y x+\cdots+K y x^{m-1}\right) y^{-1}
$$

is a direct sum. By Lemma 4.1, it follows that $u$ is left algebraic of degree $m$ over $K$. On the other hand, since $x$ is in $D^{(n)}$ and left algebraic of bounded degree $d$ over $K$, so is $u \in D^{(n)}$. Thus, again by Lemma 4.1, $m \leq d$. The proof is now complete.

\section{Acknowledgements}

The authors would like to express their sincere gratitude to the Editor and also to the referee for his/her careful reading and comments. 


\section{REFERENCES}

[1] M. Aaghabali, S. Akbari, M. H. Bien, Division Algebras with Left Algebraic Commutators, Algebr. Represent. Theor. 21 (2018) 807-816.

[2] M. Aaghabali, M. H. Bien, Certain Simple Maximal Subfields in Division Rings, Czechoslovak Mathematical Journal (accepted), arXiv:1708.08385

[3] J. P. Bell, D. Rogalski, Free subalgebras of division algebras over uncountable fields, Math. Z. 277 (2014), 591-609.

[4] J. P. Bell, V. Drensky, Y. Sharifi, Shirshov's theorem and division rings that are left algebraic over a subfield, J. Pure Appl. Algebra 217 (2013), 1605-1610.

[5] T. T. Deo, M. H. Bien, B. X. Hai, On weakly locally finite division rings, Acta Math. Vietnam. (2018), http://dx.doi.org/10.1007/s40306-018-0292-x

[6] P. M. Cohn, Quadratic extensions of skew fields, Proc. London Math. Soc. 11 (1961) 531-556.

[7] C. Faith, Algebraic Division Ring Extensions, Proc. Amer. Math. Soc. 11 (1960), 43-53.

[8] R. Hazrat, On central series of the multiplicative group of division rings, Algebra Colloq. 9 (2002), 99-106.

[9] N. Jacobson, Structure of rings, rev. ed., Amer. Math Soc. Colloq. Publ. 37, Amer. Math. Soc, Providence, R. I., 1964.

[10] T. Y. Lam, A First Course in Noncommutative Rings, in: Grad. Texts in Math., 131, Springer-Verlag, Berlin, 1991.

[11] M. Mahdavi-Hezavehi, Commutators in division rings revisited, Bull. Iran. Math. Soc. 26 (2000), 7-88.

[12] L. H. Rowen, Ring Theory, Student Edition, Academic Press, Boston, 1991.

[13] A. H. Schofield, Artin's problem for skew field extensions, Math. Proc. Camb. Phil. Soc. 97 (1985), 1-6.

[14] W. R. Scott, Group theory, Dover Publications Inc., New York, second edition, 1987.

E-mail address: bxhai@hcmus.edu.vn;trangvm8234@gmail.com; mhbien@hcmus.edu.vn

Faculty of Mathematics and Computer Science, VNuhCM-University of Science, 227 Nguyen Van Cu Str., Dist. 5, Ho Chi Minh City, Vietnam. 